\title{
RECENT RESULTS ON POLARIZED QUARK AND GLUON DISTRIBUTIONS AT COMPASS
}

\author{
I.SAVIN, JINR, DUBNA \\ On Behalf of the COMPASS Collaboration
}

The latest results of deep inelastic scattering (DIS) studies of $160 \mathrm{GeV}$ muons on the deuterated polarized target are reported. They include estimations of virtual photon-deuteron asymmetries, gluon contributions to the nucleon spin and Collins asymmetries in hadron production on the transversely polarized target.

\section{Introduction}

The COMPASS collaboration includes about 230 physicists from 30 Institutions representing 12 countries.

COMPASS stands for COmmon Muon and Proton Apparatus for Structure and Spectroscopy. It is a new fixed target facility at CERN which has been proposed [1] in 1996, constructed and put in operation in 1998-2001. Data taking has been going on since 2002.

The physics program of COMPASS consists of two parts. The first one includes studies of the nucleon spin structure by using longitudinally polarized muons and polarized targets, namely:

- determination of gluon polarization in polarized nucleons, $\Delta \mathrm{G} / \mathrm{G}$,

- measurement of transverse spin structure function $\mathrm{h}_{1}(x)$,

- updating the measurements of flavor dependent polarized quark helicity densities, $\Delta \mathrm{q}(x)$, including $\Delta \mathrm{s}(x)$,
- polarization of $\Lambda$ and $\bar{\Lambda}$ produced in deep inelastic scattering,

- vector mesons production and some other processes.

The second part of COMPASS physics concerns studies of hadron spectroscopy, using high energy hadron beams (pions, kaons, protons):

- Primakoff reactions, in particular to determine the polarizabilities of charged pions and kaons,

- $\quad$ searches for glueballs, hybrids and other exotic states,

- semileptonic charm decays,

- searches for doubly charmed baryons and other reactions.

\section{COMPASS status}

The present layout of COMPASS is shown in Fig.1.

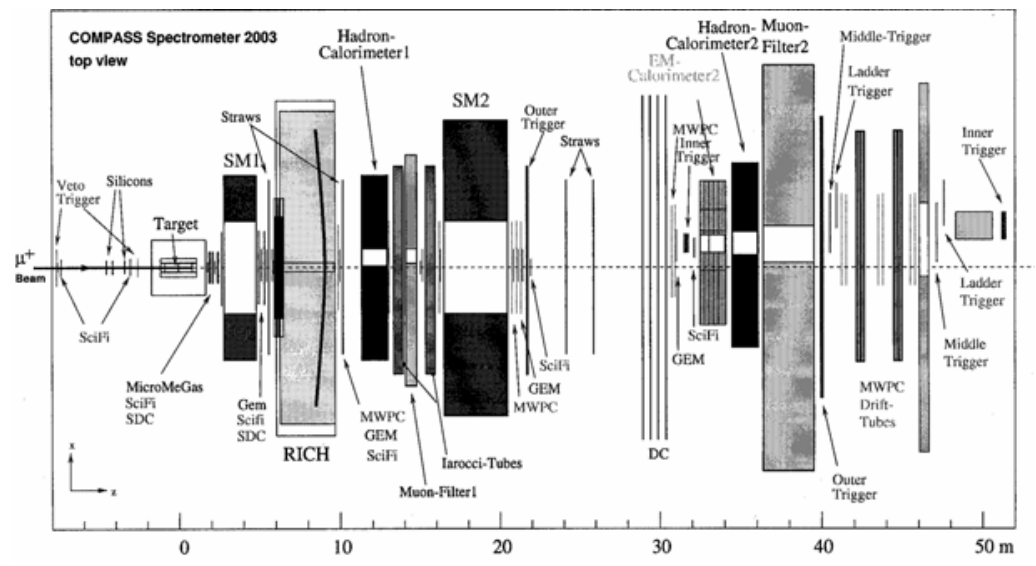

Figure 1. COMPASS Layout 
It is a two-stage spectrometer, with magnets SM1 and SM2, various trackers and particle identification detectors for secondary muons, electrons and hadrons. Many modern new technologies are used. Among others are scintillating fibers and MicroMega, GEM and straw chambers for tracking the particle trajectories, large volume calorimeters and RICH for hadron identification. The readout system is able to handle trigger rates up to $25 \mathrm{kHz}$, and data flow reach up to 4-5 Tb/day. The world largest polarized target has two $60 \mathrm{~cm}$ containers filled with ${ }^{6} \mathrm{LiD}$, which can be polarized in opposite directions either longitudinally or transversely to about $\pm 50 \%$. Simultaneous data taking from two opposite polarized targets reduces systematics in measurements of spin-target asymmetries. For 2002 to 2004 COMPASS has used a $\mu^{+}$ beam with momentum $160 \mathrm{GeV} / \mathrm{c}$, intensity $2 \cdot 10^{8} \mu /$ spill and average polarization $-76 \%$. The total sample of data accumulated on tapes in 2002 and 2003 contains about 10 billion events. The 2004 run is in progress. The broad COMPASS acceptance region, extending in $x$-Bjorken from a very small value $x \leq 10^{-5}$ to $x \sim 1$ and in $\mathrm{Q}^{2}$ from the quasireal photo-production point $\mathrm{Q}^{2} \sim 10^{-4}$ to $\mathrm{Q}^{2} \sim 100 \mathrm{GeV}^{2}$, is very well suited to study nonperturbative and perturbative QCD physics.

\section{Preliminary results}

Some preliminary results related to the spin program and based on the analysis of the 2002 data are presented here.

\subsection{Virtual photon-deuteron asymmetry $A_{1}^{d}(x)$}

This asymmetry is related to the polarized structure function $g_{1}^{d}(x)$ and has been measured before [2] by SMC, SLAC and HERMES collaborations. Potential opportunities of COMPASS, especially in the region of small $x$, are seen from the preliminary COMPASS data on $A_{1}^{d}$ obtained in 2002 (Fig.2). This sample includes about $6.510^{6}$ events within usual DIS cuts $\left(Q^{2}>1 \mathrm{GeV}^{2}\right)$. By the end of the 2004 run the statistics is expected to be 4 times larger. The final accuracy we will obtain in the low $x$ region shows COMPASS potential to improve the measurement of the first moment of $g_{1}$, and thus $\Delta \Sigma$.

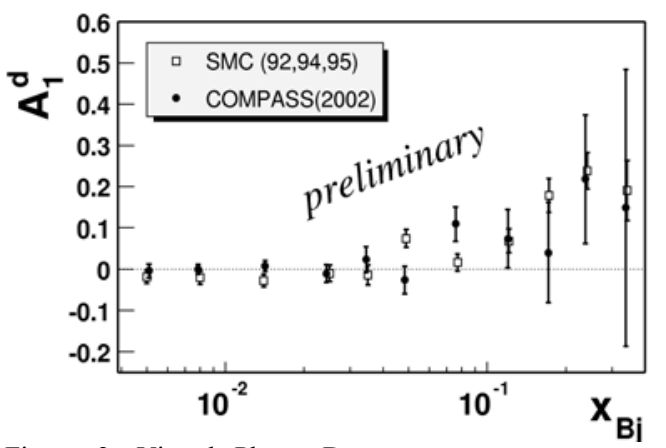

Figure 2. Virtual Photon-Deuteron asymmetry as a function of $x$-Bjorken compared to the SMC published data.

\subsection{Gluon contributions to the nucleon spin, $\Delta G / G$}

The $\Delta \mathrm{G} / \mathrm{G}$ can be measured directly from asymmetries in Semi-Inclusive Deep Inelastic Scattering (SIDIS) reactions on polarized targets induced by Photon-Gluon Fusion (PGF) processes with either open charm particles or high $\mathrm{p}_{\mathrm{t}}$ hadron pairs [1] in the final state. While the first reaction is background free, the second one is contaminated by the physics background from the processes induced by LO DIS and QCD Compton virtual photon-quark scattering. COMPASS uses both methods.

The virtual photon-deuteron asymmetry $\mathrm{A}^{\gamma^{*} \mathrm{~d}}$ for high $\mathrm{p}_{\mathrm{t}}$ pairs production evaluated from the 2002 data for all $\mathrm{Q}^{2}$, is found to be $\mathrm{A}^{\gamma^{* d} \mathrm{~d}}=-0.065 \pm 0.036$ (stat) \pm 0.010 (syst.). The systematic error is estimated so far only from experimental false asymmetries studies.

Prior to calculation of $\Delta G / G$ from $A^{\gamma^{*} d}$, one needs to subtract the physics background 
asymmetries. Physics background contaminations can be reduced by selecting events with $\mathrm{Q}^{2}>1 \mathrm{GeV}^{2}$. But this cut substantially reduces statistics. Assuming that the contribution of PGF processes to the measured asymmetry is about $1 / 4$ of the total and using all statistics expected at COMPASS by the end of 2004 run, one can determine $\Delta \mathrm{G} / \mathrm{G}$ with an accuracy $\sigma(\Delta \mathrm{G} / \mathrm{G}) \sim$ 0.05 for all $\mathrm{Q}^{2}$ and $\sim 0.17-$ for events with $\mathrm{Q}^{2}>1 \mathrm{GeV}^{2}$.

Charm reconstruction in COMPASS is demonstrated in Fig. 3 wich shows the mass peak of $D^{0}\left(\bar{D}^{0}\right) \rightarrow K \pi$ tagged by identifying $D^{*_{+}} \rightarrow \pi^{+}+D^{0}\left(K^{-} \pi^{+}\right)$and charge conjugated decays.

The projected statistics of the 2002-2004 data will allow to determine $\Delta \mathrm{G} / \mathrm{G}$ from all $\mathrm{D}$ meson decays with an accuracy of about $\sigma(\Delta \mathrm{G} / \mathrm{G}) \sim 0.25$.

\subsection{Transverse spin effects}

The complete description of the nucleon structure is known to require three structure functions: $\mathrm{f}_{1}(x)$ characterizing the momentum distributions of quarks in unpolarized

nucleons, $\mathrm{g}_{1}(x)$ characterizing helicity distributions of quarks in longitudinally polarized nucleon and $h_{1}(x)$ characterizing transversity distributions of quarks in transversely polarized nucleons. The $\mathrm{h}_{1}(x)$ can be accessed only through SIDIS reactions, in particular, by measuring the so called Collins asymmetries, $\mathrm{A}_{\text {coll }}$, in azimuthal distributions of hadrons produced in reaction $\mu+\mathrm{N} \rightarrow \mu^{\prime}+\mathrm{h}+\mathrm{X}$ on transversely polarized targets. This asymmetry has typical $\mathrm{A}(x, z) \sin \phi_{\mathrm{c}}$ form where $\phi_{\mathrm{c}}$ is a Collins angle, defined as the angle between vectors of the hadron momentum and final quark spin, and $\mathrm{A}(x, z)$ is proportional to $\mathrm{h}_{1}(x)$.

The first COMPASS results on $A_{\text {coll }}^{d}$ for negative and positive hadrons produced on deuterons, are shown in Fig.4 as a functions of $\mathrm{x}$-Bjorken and $\mathrm{z}$, the momentum fraction of the hadron $E_{h} / v$. Only the most energetic hadrons (leading hadrons) are used in the analysis, as it is the one which most probably is the hadronizations remnant of the struck quark. More data will be available for these plots from 2003 and 2004 runs.

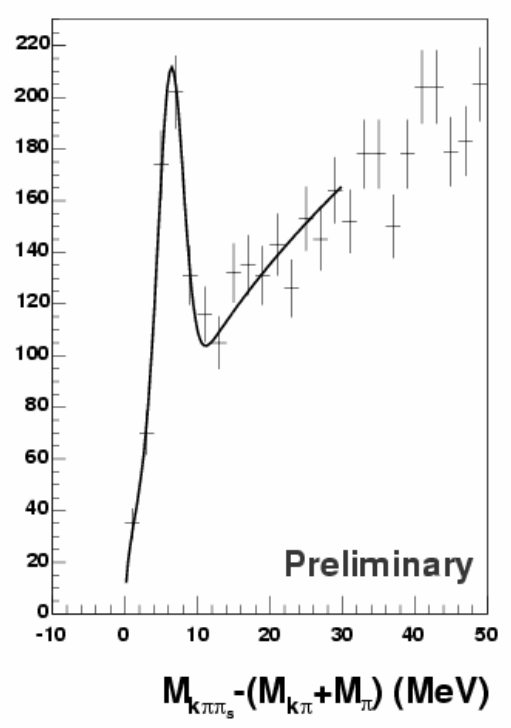

(a)

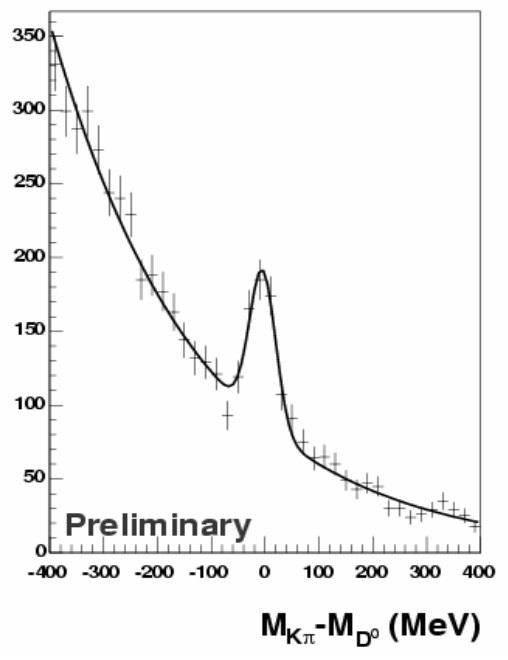

(b)

Figure 3. The mass peaks of $\mathrm{D}^{*+}$ (a) and $\mathrm{D}^{0}$ mesons (b) reconstructed at COMPASS from 2002 data sample. 

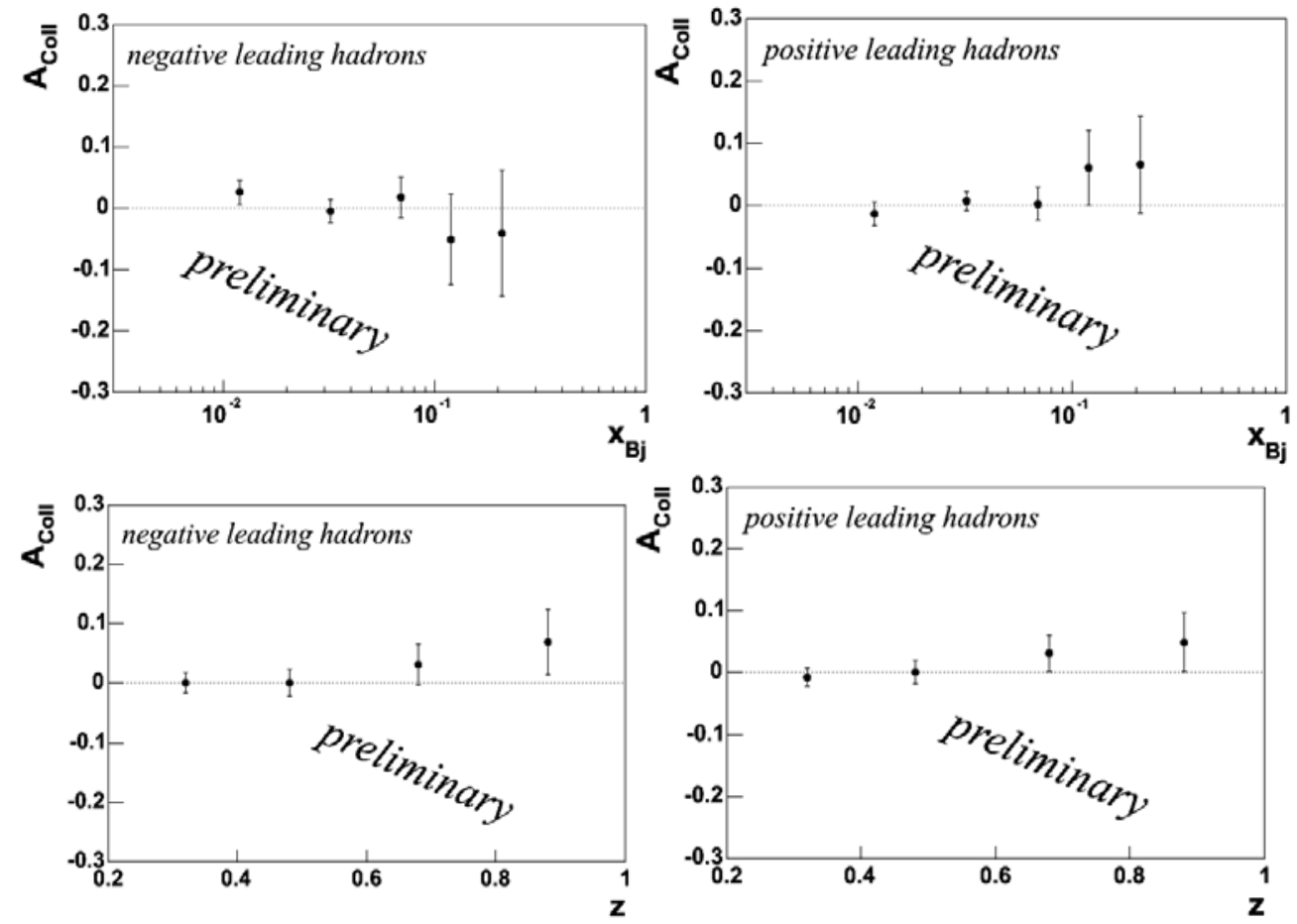

Figure 4. Collins asymmetries in production of hadrons on deuterons as a function of $x$-Bjorken and $z$.

\section{Summary}

COMPASS is a new fixed target facility at CERN started to take data in 2002. The preliminary results reported at this Conference have already shown great potential of the set up. More results will be communicated soon. Among them the most important will be the results on gluon polarization in nucleon measured directly via Photon Gluon Fusion mechanism.

\section{References}

1. COMPASS proposal: CERN/SPSLC 96-14, SPSC/P 297, March 1, 1996 and addendum, CERN/SPSLC/96-30, SPSLC/P297 Add 1, May 20, 1996.

2. SMC: B.Adeva et al, Phys.Rev.D58,112001(1998), SLAC E143: K.Abe et al., Phys.Rev.D58,112003(1998),

SLAC E155: P.L.Anthony et al., Phys.Lett.B463,339 (1999) ; Ibid D493,19(2000),

HERMES : A.Airapetian et al, Phys.Lett.B442,484(1998). 